\title{
Relação entre coeficientes de cultura e graus-dia de desenvolvimento da alface $^{1}$.
}

\author{
Elio L. da Silva ${ }^{2}$; Leôncio F. Martinez ${ }^{3}$; Muluneh Yitayew ${ }^{4}$ \\ ${ }^{2}$ UFLA -DEG, C. Postal 37, 37.200-000, Lavras-MG; ${ }^{3}$ INIA, C. Postal 36-, La Serena -Chile; ${ }^{4}$ Univ. of Arizona - ABE, 403 Shantz Bldg. \\ \#38, Tucson -Arizona 85721, USA.
}

\begin{abstract}
RESUMO
Este estudo foi conduzido na Fazenda Campbell da Universidade do Arizona , no período de junho de 1994 a fevereiro de 1995, com objetivo de determinar a equação do coeficiente de cultura $(\mathrm{Kc})$ como função de graus-dia de desenvolvimento (GDD) usando a série de senos Fourier, para alface (Lactuca sativa, L.), do tipo folhosa. Foram usadas dez parcelas casualizadas, irrigadas de maneira a manter o potencial matricial da água no solo acima ou igual a $-20 \mathrm{kPa}$, que constituiram as repetições na determinação dos valores de evapotranspiração máxima da cultura $(\mathrm{ETm})$. Cada parcela foi constituida por seis canteiros de 1,0 m x 7,3 m, com duas linhas de plantas (cultivar Waldmann's Green) em cada, com população, após desbaste, de 48 plantas por canteiro. A época de desbaste ocorreu com GDD de $228^{\circ} \mathrm{C}$ e Kc médio de 0,4 e a colheita com GDD igual a $742^{\circ} \mathrm{C}$ e Kc médio de 1,2. Os valores de Kc como função de GDD, em diferentes estádios do ciclo da cultura, permitiram gerar uma equação que pode ser inserida em programa de computador para manejo de irrigação, considerando um GDD final (GDD de ajuste) de $900^{\circ} \mathrm{C}$.
\end{abstract}

Palavras-chave: Lactuca sativa L., manejo de irrigação, evapotranspiração, AZSCHED.

\begin{abstract}
Relationship between lettuce crop coeficient and growing degree days.

This research was carried out at the Campbell Farm of The University of Arizona, from June 1994 to February 1995, with the aim of determining a growing-degree-days-based crop coefficient $(\mathrm{Kc})$ equation for leaf lettuce (Lactuca sativa $L$.), using the Fourier sine series model. Ten randomized experimental plots were irrigated, in order to maintain a soil matric potential greater or equal to -20 $\mathrm{kPa}$, as replications for the determination of crop maximum evapotranspiration (ETm). Each plot consisted of six raised beds, $7.3 \mathrm{~m}$ long and $1.0 \mathrm{~m}$ wide, with two rows of lettuce (Lactuca sativa L., cv. Waldmann's Green) thinned to a final population of 48 plants per bed. The thinning time occurred with a GDD of $228^{\circ} \mathrm{C}$ and average $\mathrm{Kc}$ of 0.4 . The harvest time occurred with GDD of $742^{\circ} \mathrm{C}$ and average $\mathrm{Kc}$ of 1.2. The values of $\mathrm{Kc}$ as function of GDD, during the crop season, allowed the generation of an equation that can be inserted into irrigation scheduling software, considering an ending GDD (adjustment GDD) of $900^{\circ} \mathrm{C}$.
\end{abstract}

Keywords: Lactuca sativa L., irrigation management, evapotranspiration, AZSCHED

\section{(Aceito para publicação em 03 de abril de 1999)}

A programação da irrigação de uma cultura consiste na determinação do momento da irrigação (quando) e da quantidade de água a ser aplicada (quanto), de maneira a compensar o consumo de água. Este consumo é função de fatores climáticos locais refletidos na evapotranspiração máxima (ETm) associada às fases fenológicas da cultura.

A evapotranspiração máxima de uma cultura é geralmente determinada, de maneira indireta, pela sua relação com a evapotranspiração potencial de uma cultura de referência (ETo), através dos coeficientes de cultura $(\mathrm{Kc}),\left(\mathrm{ETc}=\mathrm{ETo}^{\text {' }}\right.$ $\mathrm{Kc}$ ). Os valores de $\mathrm{Kc}$ refletem as necessidades hídricas das plantas nos seus estágios vegetativos e reprodutivos. Vários métodos podem ser usados na estimativa da evapotranspiração de referência.
Segundo Cuenca (1989), o método de Penman, ou os seus derivados, por envolverem um maior número de parâmetros, tem sido um dos mais recomendados. É um dos métodos mais utilizados em todo o mundo para estimativa de ETo (Jensen et al., 1990).

As fases, ou estádios da cultura, fator de grande importância na determinação do consumo de água, podem ser definidas de diversas formas, tais como: dias após plantio, dias após emergência, índice de área foliar e graus-dia de desenvolvimento (GDD). Tradicionalmente, os coeficientes Kc têm sido determinados com base em semana ou mês do ano. Pesquisas recentes têm sido desenvolvidas com objetivo de se determinar coeficientes de cultura como função do estágio fenológico, percentagem do ci- clo (Tosso \& Torres, 1986), e graus-dia de desenvolvimento (Sammis et al.,1985; Fox et al., 1992).

Os coeficientes de cultura apresentados, para as fases fenológicas ou para o ciclo da cultura, em relação ao número de dias após o plantio, podem levar à estimativa equivocada de demanda de água em regiões diferentes, devido à variação no ciclo de desenvolvimento imposta pelos fatores climáticos, principalmente, a temperatura.

$\mathrm{O}$ aumento de temperatura acelera o desenvolvimento da planta, reduzindo o seu ciclo (Chandler, citado por Infeld \& Silva, 1987). Com base nesse princípio ficavam explicadas as diferentes durações do ciclo de uma cultura, em dias, para cultivos em localidades com regimes de temperaturas diferentes. A

\footnotetext{
${ }^{1}$ Parte da tese de doutorado do primeiro autor apresentada à "The University of Arizona". Trabalho executado com apoio financeiro do CNPq.
} 
maioria dos processos fisiológicos nas plantas superiores ocorrem entre temperaturas de 0 a $40^{\circ} \mathrm{C}$ e apesar dessa ampla faixa de temperatura, o melhoramento genético tem trabalhado na busca de ampliar esse intervalo.

Vários trabalhos têm demonstrado a grande utilidade do uso GDD para previsão das fases fenológicas, bem como zoneamento de culturas (Gilmore \& Rogers,1958; Sammis et al.,1985; Slack et al.,1994). Esta forma de "quantificar" as fases fenológicas da planta, tem como característica o fato de que GDD independe da época e do local do plantio. Estes trabalhos de pesquisas têm demonstrado uma maior adaptação do ciclo de algumas culturas ao GDD do que à quantidade de dias do calendário. Gilmore \& Rogers (1958) trabalhando com milho híbrido, demonstraram a eficiência do GDD para prever embonecamento do milho em lugar da variação de dias. Sammis et al.(1985) afirmam que um contador de tempo ou "relógio fisiológico" desenvolvido com base em GDD é menos variável do que a contagem por dias do calendário.

O conceito de graus-dia ou unidades térmicas, pressupõe a existência de uma temperatura abaixo da qual a planta não se desenvolve ou, se o fizer, o faz a uma taxa muito reduzida, denominada temperatura base. Esse conceito se baseia no fato de que uma planta necessita de certa quantidade de energia, representada pela soma de ${ }^{\circ} \mathrm{C}, \mathrm{GDD}$, acima do valor da temperatura-base, para completar determinada fase fenológica ou, mesmo, seu ciclo total.

Os estudos das interações climaplanta foram iniciados, segundo Mota (1986), por Reámur, em 1735, sendo este considerado o precursor do sistema de graus-dia ou unidades térmicas, GD. Um grau-dia, ou unidade térmica, GD, era definido como a temperatura média do dia. No método original aplicado por Réaumur, a constante térmica era calculada a partir da soma das temperaturas médias diárias acima de $0^{\circ} \mathrm{C}$, que podia ser determinada para o ciclo total ou para cada fase. Esse método foi denominado de método direto, que apresentava o inconveniente de sofrer variações segundo as localidades consideradas (Mota, 1986). De acordo com este autor, para atender ao cálculo de grausdia para diversas localidades deve-se usar o método residual, que consiste no somatório das diferenças entre a temperatura média diária e a temperatura base.

A planta apresenta uma faixa com pontos extremos, inferior e superior, de temperatura abaixo e acima dos quais ela praticamente cessa o seu crescimento e desenvolvimento, ou continua a taxas relativamente reduzidas. Esses valores são denominados de "temperaturas cardeais". A complexidade fisiológica da planta impede a determinação precisa destas temperaturas. Contudo, é possível encontrar na literatura resultados de pesquisas dando faixas de valores aproximados das temperaturas ótimas e extremas, nas quais espera-se um pleno desenvolvimento da espécie cultivada. $\mathrm{O}$ sucesso do uso de GDD para programação e manejo de irrigação se baseia na informação das temperaturas cardeais. Madariaga \& Knott (1951) e Slack et al. (1994) sugerem os valores $4,4^{\circ} \mathrm{C}$ e $21,1^{\circ} \mathrm{C}$ como as temperaturas cardeais da alface.

Sammis et al. (1985) descobriram que um polinômio de terceira ordem descreve muito bem a relação entre coeficiente de cultura e graus-dias de desenvolvimento para alfafa, milho, sorgo, e algodão. A mesma metodologia foi usada por Plant et al. (1992) em algodão. Shayya et al. (1991) encontraram uma relação linear entre Kc e GDD para milho. Uma metodologia diferente foi usada por Fox Jr. et al. (1992) que usaram séries de Fourier para descrever Kc com base em GDD, a partir de valores de ETm fornecidos por Erie et al. ( 1982) para várias culturas.

Este trabalho teve por objetivo estabelecer uma relação entre o desenvolvimento da cultura da alface e sua evapotranspiração máxima, para diferentes épocas e locais de plantio, para determinar a equação do coeficiente de cultura $(\mathrm{Kc})$ como função de graus-dia de desenvolvimento, usando a série de senos Fourier.

\section{MATERIAL E MÉTODOS}

O estudo foi desenvolvido na Fazenda Campbell, da Universidade do Arizona, no período de junho de 1994 a fevereiro de 1995, com o último plantio efetuado em 09 de novembro de 1994. O local é de clima semi-árido, em latitude $32^{\circ} 16^{\prime} 49^{\prime \prime} \mathrm{N}$, longitude $111^{\circ} 58^{\prime} 16^{\prime \prime} \mathrm{W}$ e a 713 metros acima do nível médio do mar. A análise textural indicou solo composto por $51 \%$ de areia, $38 \%$ de silte e $11 \%$ de argila (Silva, 1995).

Estudou-se a alface australiana, variedade "Waldman's Green", do tipo folhosa, em dez parcelas, sendo cinco irrigadas por gotejamento subsuperficial (parcelas A, B, C, D e E) e outras cinco parcelas (F, G, H, I e J) irrigadas por sulcos curtos e nivelados. Comparando o efeito de métodos de irrigação, em outro estudo com o mesmo experimento, Silva (1995) conclui que não houve diferença significativa entre as parcelas irrigadas por sulcos e aquelas irrigadas por gotejamento, no que se refere a evapotranpiração. $\mathrm{O}$ momento de irrigação foi definido com uso de tensiômetros instalados a $10,15,20,25$ e 30 centimetros de profundidades. Usou-se a tensão média tomada nas cinco profundidades, de aproximadamente $-20 \mathrm{kPa}$, conforme estudos por Taylor (1965), Sammis (1980) e Sammis et al. (1988). A quantidade de água aplicada foi tal a repor cem por cento do consumo ocorrido entre duas irrigações consecutivas.

Determinou se a evapotranspiração da cultura nas dez parcelas, aleatoriamente distribuidas numa área experimental de aproximadamente $1.250 \mathrm{~m}^{2}$. Cada parcela consistiu de seis canteiros 1,0 m x 7,3 m cada, com duas fileiras de plantas por canteiro. $\mathrm{O}$ espaçamento usado foi de $0,30 \mathrm{~m}$ entre fileiras e entre plantas no canteiro, representando uma densidade populacional de 6.500 plantas por hectare.

Instalou-se, no centro de cada parcela uma bateria de tensiômetros com as profundidades de 5, 10, 15, 20, 25, 45 e $55 \mathrm{~cm}$. Os valores de potencial matricial da água no solo foram obtidos diariamente através de tensímetro digital portátil. Os cinco primeiros tensiômetros foram usados para a determinação da tensão média da água na camada 0 a $30 \mathrm{~cm}$ do solo. Os dois últimos tensiômetros foram usados para determinação do gradiente de potencial matricial a $50 \mathrm{~cm}$ de profundidade pela equação 7. O conteúdo de água médio do solo na camada de 0 a $50 \mathrm{~cm}$, foi 
determinado pelo método da reflectometria de onda eletromagnética (TDR ,"Time Domain Reflectometer”), com medidas em todas as parcelas. Usou-se a equação de calibração do TDR, para o solo estudado, determinada por Silva (1995):

\section{(Equação 1)}

em que $\mathrm{Y}=$ umidade do solo expressa em base de volume $\left(\mathrm{cm}^{3} \mathrm{~cm}^{-3}\right)$ e $\mathrm{X}=$ umidade do solo, com base em volume, dada pelo instrumento.

A evapotranspiração de referência, ETo, foi estimada pela equação de Penman inicialmente modificada por Pruitt (Doorenbos \& Pruitt, 1977) conhecida como equação Penman-FAO, dada por:

\section{(Equação 2)}

Em que $\mathrm{c}=$ coeficiente de ajuste, baseado em dados climatológicos;

$\Delta \quad=$ fator de ponderação depen-

$\overline{\Delta+\gamma}$ dente da temperatura e altitude

$\Delta=$ derivada da pressão de saturação de vapor como função da temperatura, para $\mathrm{T}=$ temperatura média em ${ }^{\circ} \mathrm{C}$; $\mathrm{g}=$ constante psicrométrica, $\mathrm{mb}^{\circ} \mathrm{C}^{-1} ; \mathrm{Rn}$ = radiação líquida, $m m \cdot \operatorname{dia}^{-1} ; \mathrm{G}=$ fluxo de calor para o solo, mm.dia ${ }^{-1} ; \mathrm{U}_{2 \mathrm{~m}}=$ velocidade do vento a 2 metros de altura, km.dia ${ }^{-1} ; e_{\mathrm{s}}=$ pressão de saturação de vapor do ar, $\mathrm{kPa} ; \mathrm{e}_{\mathrm{a}}=$ pressão real de vapor do ar, $\mathrm{kPa}$.

Fervert et al. (1983) expressaram o coeficiente de ajuste (c) em forma de tabela. Mais tarde, Cuenca \& Jensen(1988), citados por Cuenca (1989), expressaram os valores de c por:

(Equação 3)

em que $\mathrm{RH}_{\max }=$ umidade relativa máxima do ar, \%; $\mathrm{R}_{\mathrm{s}}=$ radiação solar expressa em equivalente de evaporação, mm.dia ${ }^{-1} ;$ Udia $=$ velocidade média do vento, durante o dia, a $2 \mathrm{~m}$ de altura, $\mathrm{m}$ $\mathrm{s}^{-1} ; \mathrm{U}_{\mathrm{dia}} / \mathrm{U}_{\text {noite }}=$ relação entre velocidade média do vento durante o dia e durante a noite (dia considerado arbitrariamente como 7:00 as 19:00 horas)

Um exemplo de determinacao passo a passo da evapotranspiração de referência pelo método de Penman-FAO pode ser encontrado em Cuenca (1989).

A evapotranspiração máxima (ETm) da alface foi obtida a partir de medições,

\section{Equação 1}

$\mathrm{Y}=0,714 \mathrm{X}+4,483\left(\mathrm{r}^{2}=0,9383\right)$

\section{Equação 2}

$\mathrm{ETo}=\mathrm{c}\left[\frac{\Delta}{\Delta+\gamma}\left(\mathbf{R}_{\mathbf{n}}-\mathrm{G}\right)+\frac{\gamma}{\Delta+\gamma} \cdot 2,7\left(1,0+0,01 \mathrm{U}_{2 \mathrm{~m}}\right)\left(\mathrm{e}_{\mathrm{s}}-\mathrm{e}_{\mathbf{a}}\right)\right]$

\section{Equação 3}

$\mathrm{c}=0,68+0,0028\left(\mathrm{RH}_{\max }\right)+0,018\left(\mathrm{R}_{\mathrm{s}}\right)-0,068\left(\mathrm{U}_{\text {dia }}\right)+0,013\left(\mathrm{U}_{\text {dia }} / \mathrm{U}_{\text {noite }}\right)+0,0097$ $\left(\mathrm{U}_{\text {dia }}\right)\left(\mathrm{U}_{\text {dia }} / \mathrm{U}_{\text {noite }}\right)+0,43 \times 10^{-4}\left(\mathrm{RH}_{\max }\right)\left(\mathrm{R}_{\mathrm{s}}\right)\left(\mathrm{U}_{\text {dia }}\right)$

\section{Equação 4}

$$
\int_{\mathrm{t} i}^{\mathrm{t}}\left[(\mathrm{p}+\mathrm{i}+\mathrm{r})-\mathrm{e}-\mathrm{d} \mathrm{z}_{\mathrm{z}}\right] \mathrm{dt}=\int_{\mathrm{t}}^{\mathrm{t}} \int_{0}^{\mathrm{z}} \frac{\partial \theta}{\partial \mathrm{t}} \mathrm{d} \mathrm{zdt}
$$

\section{Equação 5}

$\mathrm{P}+\mathrm{I}+\mathrm{ES}=\mathrm{ET} \pm_{[\mathrm{CB} 1]} \mathrm{D} \pm \Delta \mathrm{A}$

em campo, pelo metodo do balanço hídrico do solo. A equação geral que descreve o balanço de água na camada superficial do solo é apresentada por Slatyer (1970) como:

\section{(Equação 4)}

em que $\left(t_{\mathrm{f}}-\mathrm{t}_{\mathrm{i}}\right)$ corresponde ao intervalo de tempo entre as medições. $\mathrm{O}$ termo $p$ representa as taxas de precipitação pluviométrica efetivas, assumidas como sendo taxas de precipitação pluviométrica de baixa intensidade, ou seja, intensidade menor que a infiltrabilidade do solo. O termo $i$ (irrigação) foi obtido através da irrigação controlada. O termo $r$ (escoamento superficial ou runoff) foi considerado negligível no presente estudo uma vez que a superfície do solo apresentava declividade zero e as chuvas ocorridas foram de baixa intensidade. O termo $e$ representa a evapotranspiração e $d_{\mathrm{z}}$ a drenagem. q é a umidade do solo expressa com base em volume $\left(\mathrm{cm}^{3} \mathrm{~cm}^{-3}\right)$.
Jury et al. (1991) apresentam a equação do balanço hídrico no solo, para intervalo definido de tempo,como:

(Equação 5)

onde os termos do lado esquerdo representam a precipitação pluviométrica efetiva, P, irrigação, I, e escoamento superficial, ES. A soma destes termos representam a entrada liquida de água no perfil de solo num determinado periodo de tempo de interesse. No lado direito da equação estão evapotranspiração, ET, drenagem profunda, D, e variação de armazenamento no perfil de solo, DA. Todos os termos da equação são positivos com excessão de D e DA que podem ser positivo ou negativo. Um valor negativo de $\mathrm{D}$ representa fluxo ascendente de água vindo da camada abaixo da profundidade até onde o balanço é conduzido. Variação negativa de armazenamento implica em valores finais menores do que os iniciais. 
A saída (drenagem) ou entrada de água (ascenção) na camada de solo em estudo, (D), foi determinada com uso da equação, expressa por Reichardt (1985),

(Equação 6)

onde $\mathrm{q}_{\mathrm{z}}$ é a densidade de fluxo calculada usando a "lei de DarcyBuckinghan" (Cassel \& Nielsen, 1986) que pode ser expressa por

(Equação 7)

em que $\mathrm{K}(\mathrm{h})$ =condutividade hidráulica como função do potencial matricial; $\frac{\partial \mathrm{h}}{\partial \mathrm{z}}=\mathrm{o}$ gradiente de potencial matricial; $\mathrm{Z}=$ profundidade.

Os valores de $\frac{\partial \mathrm{h}}{\partial \mathrm{z}}$, gradiente de potencial matricial, foram aproximados pela média do gradiente de potencial matricial no inicio (i) e no final (f) de um dado periodo, isto é,

(Equação 8)

em que $h_{c}=$ potencial matricial da água no solo na profundidade $\mathrm{z}_{\mathrm{c}}$ (ponto de cima) $\mathrm{h}_{\mathrm{b}}=$ potencial matricial $\mathrm{da}$ água no solo na profundidade $\mathrm{z}_{\mathrm{b}}$ (ponto de baixo); i, f = índices que indicam instante inicial e final, respectivamente

Usou se a expressão de Gardner da condutividade hidráulica (K) como função do potencial matricial (h), segundo Reichardt (1985),

(Equação 9)

onde K' e a são os parâmetros de ajuste especificos para a camada de solo. Para o solo do experimento, foi determinado $\mathrm{K}^{\prime}=0,00123 \mathrm{~cm} \mathrm{~s}^{-1} \mathrm{e}$ a $=0,0501$ $\mathrm{cm}^{-1}$ por Silva (1995) e Khan (1994).

Medidas de tensão e umidade foram tomadas em intervalos de tempo variáveis ao longo do ciclo de crescimento da cultura. Usou-se intervalo de dois dias quando não ocorria chuva ou irrigação. Devido à impossibilidade de caminhar entre os canteiros após uma chuva, os intervalos estudados foram muitas vezes maiores que dois dias.

A equação 5 foi portanto usada para determinação da evapotranspiração máxima da cultura (ETm), uma vez que, todos os demais parâmetros daquela equação podiam ser avaliados.

As determinações de graus-dia (GD) e Graus-Dia de Desenvolvimento (GDD) foram feitas de acordo com Snyder (1985), com temperaturas base e superior para a alface dadas por Madariaga \& Knott (1951) e Slack et al. (1994) considerando-se quatro casos:

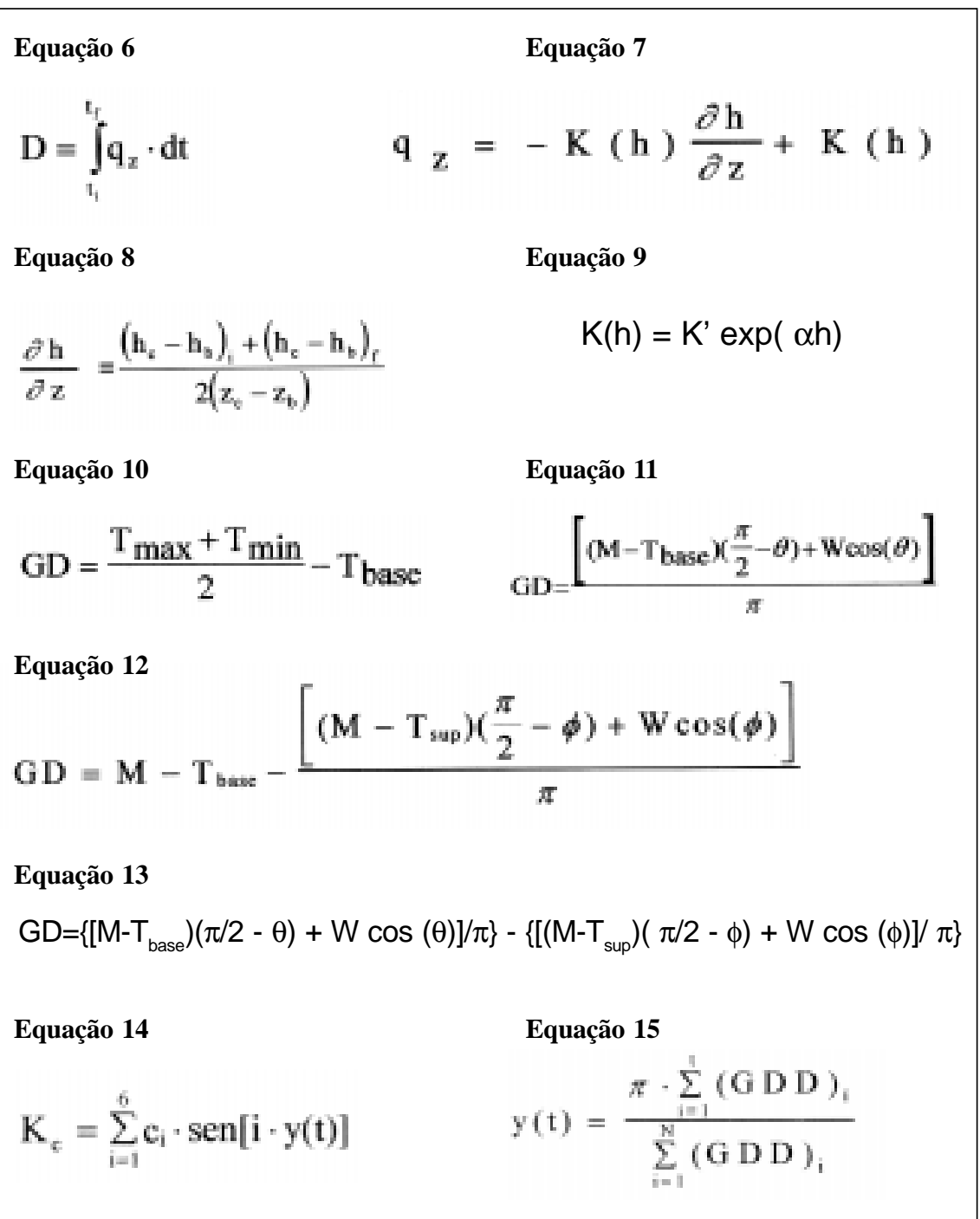

a) Temperatura mínima do dia acima da base e temperatura máxima do dia abaixo do limite superior. Para este caso, os valores de GD são determinados como:

(Equação 10)

em que GD = graus-dia, ${ }^{\circ} \mathrm{C} ; \mathrm{T}_{\max }=$ temperatura máxima do ar, ${ }^{\circ} \mathrm{C} ; \mathrm{T}_{\min }=$ temperatura mínima do ar, ${ }^{\circ} \mathrm{C} ; \mathrm{T}_{\text {base }}=$ temperatura base para a planta, ${ }^{\circ} \mathrm{C}$

b) Temperatura mínima do dia abaixo da base e temperatura máxima do dia abaixo do limite superior. Para este caso, os valores de GD são determinados como:

(Equação 11)

em que $\theta=\operatorname{arcsen}\left[\left(\mathrm{T}_{\text {iqe }}-\mathrm{M}\right) / \mathrm{W}\right] ; \mathrm{M}=$ $\left(\mathrm{T}_{\max }+\mathrm{T}_{\text {min }}\right) / 2 ; \mathrm{W}=\left(\mathrm{T}_{\max }-\mathrm{T}_{\text {min }}\right) / 2$

c) Temperatura mínima do dia acima da base e temperatura máxima do dia acima do limite superior. Para este caso, os valores de GD são determinados como:

(Equação 12) em que $\phi=\operatorname{arcsen}\left[\left(\mathrm{T}_{\text {sup }}-\mathrm{M}\right) / \mathrm{W}\right]$;

d) Temperatura mínima do dia abaixo da base e temperatura máxima do dia acima do limite superior. Para este caso, os valores de GD são determinados como:

(Equação 13)

GDD e Kc foram ajustados usando regressão linear multipla, de maneira a determinar os coeficientes $c_{\mathrm{i}}$ da série seno de Fourier, representada pela Equação 14, tendo os valores de coeficientes de cultura como variável dependente versus até seis termos $\{\operatorname{sen}[\mathrm{i} \mathrm{y}(\mathrm{t})]\}$, como variáveis independentes.

(Equação 14)

A variável $\mathrm{y}(\mathrm{t})$ foi determinada segundo Fox Jr. et al. (1992) que definiram esta variável como função de GDD a partir do plantio, transplantio, ou emergência, até um dia $t$ onde o coeficiente de cultura teria um valor igual a zero, conforme Equação 15. 
Tabela 1. Resumo do balanço hídrico do solo para determinação de ETm, valores médios de Eto calculados pela equação Penman-FAO e valores calculados de Kc para cada parcela de estudo. Tucson-AZ, 1994-1995.

\begin{tabular}{|c|c|c|c|c|c|c|c|c|c|c|c|}
\hline Período & Parcela & $\begin{array}{l}\text { h médio } \\
\text { cm.c.a. }\end{array}$ & $\begin{array}{l}\text { grad. } h \\
\mathrm{~cm} / \mathrm{cm}\end{array}$ & $\begin{array}{c}\mathrm{K} \\
\mathrm{cm} / \mathrm{h}\end{array}$ & $\begin{array}{c}\text { fluxo (q) } \\
\mathrm{cm} / \mathrm{h}\end{array}$ & $\begin{array}{c}\mathrm{D} \\
\mathrm{cm}\end{array}$ & $\begin{array}{c}\mathrm{I} \\
\mathrm{cm}\end{array}$ & $\begin{array}{l}\Delta \mathrm{A} \\
\mathrm{cm}\end{array}$ & $\begin{array}{c}\mathrm{ETm} \\
\mathrm{mm} / \mathrm{dia}\end{array}$ & $\begin{array}{c}\text { ETo } \\
\mathrm{mm} / \mathrm{dia}\end{array}$ & Kc \\
\hline \multirow[t]{5}{*}{ 10-12/Dez. } & A & $-119,54$ & 0,34 & 0,0078 & 0,0052 & 0,26 & 0 & $-0,54$ & 1,41 & 2,89 & 0,49 \\
\hline & B & $-111,63$ & 1,51 & 0,0117 & $-0,0059$ & $-0,30$ & 0 & 0,20 & 0,50 & 2,89 & 0,17 \\
\hline & D & $-112,40$ & $-0,99$ & 0,0112 & 0,0223 & 1,12 & 0 & $-1,56$ & 2,20 & 2,89 & 0,76 \\
\hline & G & $-103,72$ & 0,34 & 0,0173 & 0,0115 & 0,57 & 0 & $-0,82$ & $1, .25$ & 2,89 & 0,43 \\
\hline & 1 & $-107,55$ & $-0,94$ & 0,0143 & 0,0277 & 1,39 & 0 & $-1,59$ & 1,00 & 2,89 & 0,35 \\
\hline \multirow[t]{10}{*}{ 12-14/Dez. } & A & $-136,90$ & 0,34 & 0,0033 & 0,0022 & 0,10 & 0 & $-0,29$ & 0,95 & 2,06 & 0,46 \\
\hline & B & $-132,30$ & 1,36 & 0,0041 & $-0,0015$ & $-0,07$ & 0 & 0,01 & 0,30 & 2,06 & 0,15 \\
\hline & C & $-137,4$ & $-0,07$ & 0,0032 & 0,0034 & 0,16 & 0 & $-0,11$ & $-0,25$ & 2,06 & - \\
\hline & D & $-128,22$ & $-0,89$ & 0,0051 & 0,0096 & 0,46 & 0 & $-0,63$ & 0,86 & 2,06 & 0,42 \\
\hline & E & $-120,3$ & $-0,94$ & 0,0075 & 0,0146 & 0,70 & 0 & $-0,83$ & 0,65 & 2,06 & 0,32 \\
\hline & $\mathrm{F}$ & $-134,3$ & $-0,07$ & 0,0037 & 0,0040 & 0,19 & 0 & $-0,29$ & 0,51 & 2,06 & 0,25 \\
\hline & G & $-115,46$ & 0,85 & 0,0096 & 0,0015 & 0,07 & 0 & $-0,56$ & 2,45 & 2,06 & 1,19 \\
\hline & $\mathrm{H}$ & $-148,6$ & 0,54 & 0,0018 & 0,0008 & 0,04 & 0 & $-0,28$ & 1,19 & 2,06 & 0,58 \\
\hline & 1 & $-127,45$ & $-0,53$ & 0,0053 & 0,0081 & 0,39 & 0 & $-0,56$ & 0,85 & 2,06 & 0,41 \\
\hline & J & $-147,4$ & $-1,45$ & 0,0019 & 0,0048 & 0,23 & 0 & $-0,36$ & 0,65 & 2,06 & 0,32 \\
\hline \multirow[t]{10}{*}{ 14-16/Dez. } & A & $-149,14$ & 0,54 & 0,0018 & 0,0008 & 0,04 & 0 & $-0,23$ & 0,96 & 2,38 & 0,40 \\
\hline & B & $-147,36$ & 1,31 & 0,0019 & $-0,0006$ & $-0,03$ & 0 & $-0,16$ & 0,95 & 2,38 & 0,40 \\
\hline & C & $-144,6$ & $-0,17$ & 0,0022 & 0,0026 & 0,13 & 0 & $-0,31$ & 0,90 & 2,38 & 0,38 \\
\hline & D & $-140,21$ & $-0,33$ & 0,0028 & 0,0037 & 0,18 & 0 & $-0,21$ & 0,15 & 2,38 & 0,06 \\
\hline & E & $-134,3$ & $-0,48$ & 0,0037 & 0,0055 & 0,27 & 0 & $-0,54$ & 1,35 & 2,38 & 0,57 \\
\hline & $\mathrm{F}$ & $-145,8$ & 0,18 & 0,0021 & 0,0017 & 0,08 & 0 & $-0,17$ & 0,45 & 2,38 & 0,19 \\
\hline & G & $-124,90$ & 1,00 & 0,0060 & 0,0000 & 0,00 & 0 & $-0,20$ & 0,99 & 2,38 & 0,42 \\
\hline & $\mathrm{H}$ & $-154,0$ & 0,59 & 0,0014 & 0,0006 & 0,03 & 0 & $-0,11$ & 0,41 & 2,38 & 0,17 \\
\hline & 1 & $-139,70$ & $-0,02$ & 0,0029 & 0,0029 & 0,14 & 0 & $-0,54$ & 1,98 & 2,38 & 0,83 \\
\hline & $\mathrm{J}$ & $-154,8$ & $-2,01$ & 0,0013 & 0,0040 & 0,19 & 0 & $-0,36$ & 0,85 & 2,38 & 0,36 \\
\hline \multirow[t]{10}{*}{ 16-20/Dez. } & A & $-145,32$ & 0,39 & 0,0022 & 0,0013 & 0,13 & 1,2 & 0,52 & 1,38 & 3,01 & 0,46 \\
\hline & B & $-138,17$ & 0,80 & 0,0031 & 0,0006 & 0,06 & 1,2 & 0,42 & 1,80 & 3,01 & 0,60 \\
\hline & C & $-155,8$ & 0,03 & 0,0013 & 0,0012 & 0,12 & 1,2 & 0,65 & 1,08 & 3,01 & 0,36 \\
\hline & D & $-139,59$ & $-0,04$ & 0,0029 & 0,0030 & 0,29 & 1,2 & 0,21 & 1,76 & 3,01 & 0,58 \\
\hline & E & $-143,8$ & $-0,43$ & 0,0023 & 0,0033 & 0,32 & 1,2 & 0,44 & 1,10 & 3,01 & 0,37 \\
\hline & $\mathrm{F}$ & $-154,0$ & 0,39 & 0,0014 & 0,0009 & 0,08 & 1,2 & 0,67 & 1,13 & 3,01 & 0,38 \\
\hline & G & $-116,74$ & 1,00 & 0,0090 & 0,0000 & 0,00 & 1,2 & 0,71 & 1,23 & 3,01 & 0,41 \\
\hline & $\mathrm{H}$ & $-160,9$ & 0,64 & 0,0010 & 0,0004 & 0,03 & 1,2 & 0,86 & 0,78 & 3,01 & 0,26 \\
\hline & 1 & $-133,88$ & 0,97 & 0,0038 & 0,0001 & 0,01 & 1,2 & 0,63 & 1,40 & 3,01 & 0,47 \\
\hline & $\mathrm{J}$ & $-166,2$ & $-1,45$ & 0,0008 & 0,0019 & 0,18 & 1,2 & 0,55 & 1,18 & 3,01 & 0,39 \\
\hline
\end{tabular}

O denominador da Equação 15 representa, portanto, o total acumulado de graus-dia de desenvolvimento no final do ciclo da cultura e o numerador corresponde ao valor de GDD acumulado até um dia $t$ do ciclo vegetativo, na fase fenológica de interesse.

\section{RESULTADOS E DISCUSSÃO}

Os valores médios de ETo determinados pela equação de Penman-FAO são apresentados na Tabela 1. Observa-se uma tendência de crescimento dos valo- res de ETo obtidos durante o período de estudo, embora tenha ocorrido, frequentemente, redução de um dia para o outro. Como os fatores climáticos envolvidos na determinação de ETo, tais como, umidade relativa do ar, temperatura e radição solar, foram obtidos dentro do experi- 
Tabela 1. (Continuação)

\begin{tabular}{|c|c|c|c|c|c|c|c|c|c|c|c|}
\hline Período & Parcela & $\begin{array}{l}\mathrm{h} \text { médio } \\
\text { cm.c.a. }\end{array}$ & $\begin{array}{l}\text { grad. } \mathrm{h} \\
\mathrm{cm} / \mathrm{cm}\end{array}$ & $\begin{array}{c}\mathrm{K} \\
\mathrm{cm} / \mathrm{h}\end{array}$ & $\begin{array}{c}\text { fluxo (q) } \\
\mathrm{cm} / \mathrm{h}\end{array}$ & $\begin{array}{c}D \\
\mathrm{~cm}\end{array}$ & $\begin{array}{c}\mathrm{I} \\
\mathrm{cm}\end{array}$ & $\begin{array}{l}\Delta \mathrm{A} \\
\mathrm{cm}\end{array}$ & $\begin{array}{c}\mathrm{ETm} \\
\mathrm{mm} / \mathrm{dia}\end{array}$ & $\begin{array}{c}\text { ETo } \\
\mathrm{mm} / \mathrm{dia}\end{array}$ & Kc \\
\hline \multirow[t]{10}{*}{ 20-24/Dez. } & A & $-147,36$ & 0,08 & 0,0019 & 0,0018 & 0,17 & 0 & $-0,62$ & 1,13 & 2,81 & 0,40 \\
\hline & B & $-138,17$ & 0,69 & 0,0031 & 0,0009 & 0,09 & 0 & $-0,60$ & 1,28 & 2,81 & 0,46 \\
\hline & C & $-167,3$ & 0,59 & 0,0007 & 0,0003 & 0,03 & 0 & $-0,27$ & 0,60 & 2,81 & 0,21 \\
\hline & D & $-142,30$ & 0,09 & 0,0021 & 0,0019 & 0,18 & 0 & $-0,68$ & 1,25 & 2,81 & 0,44 \\
\hline & E & $-155,5$ & $-0,23$ & 0,0013 & 0,0016 & 0,15 & 0 & $-0,56$ & 1,03 & 2,81 & 0,37 \\
\hline & $\mathrm{F}$ & $-162,2$ & 0,59 & 0,0009 & 0,0004 & 0,04 & 0 & $-0,27$ & 1,16 & 2,81 & 0,41 \\
\hline & G & $-116,74$ & 0,69 & 0,0090 & 0,0028 & 0,27 & 0 & $-0,66$ & 0,98 & 2,81 & 0,35 \\
\hline & $\mathrm{H}$ & $-175,2$ & 0,85 & 0,0005 & 0,0001 & 0,01 & 0 & $-0,34$ & 0,83 & 2,81 & 0,30 \\
\hline & 1 & $-134,65$ & 0,19 & 0,0037 & 0,0030 & 0,29 & 0 & $-0,79$ & 1,24 & 2,81 & 0,44 \\
\hline & $J$ & $-184,1$ & $-1,45$ & 0,0003 & 0,0008 & 0,07 & 0 & $-0,55$ & 1,20 & 2,81 & 0,43 \\
\hline \multirow[t]{10}{*}{ 09-13/Jan. } & A & $-138,43$ & $-0,28$ & 0,0030 & 0,0039 & 0,37 & 0 & $-0,99$ & 1,56 & 2,63 & 0,59 \\
\hline & B & $-112,65$ & 1,10 & 0,0111 & $-0,0011$ & $-0,11$ & 0 & $-0,36$ & 1,18 & 2,63 & 0,45 \\
\hline & C & $-148,4$ & 0,29 & 0,0018 & 0,0013 & 0,13 & 0 & $-0,87$ & 1,86 & 2,63 & 0,71 \\
\hline & D & $-139,70$ & $-0,12$ & 0,0029 & 0,0032 & 0,31 & 0 & $-1,33$ & 2,55 & 2,63 & 0,97 \\
\hline & E & $-127,7$ & $-0,28$ & 0,0052 & 0,0067 & 0,64 & 0 & $-1,32$ & 1,70 & 2,63 & 0,65 \\
\hline & $\mathrm{F}$ & $-136,9$ & $-0,28$ & 0,0033 & 0,0042 & 0,40 & 0 & $-0,97$ & 1,43 & 2,63 & 0,54 \\
\hline & G & $-133,58$ & $-0,94$ & 0,0039 & 0,0075 & 0,72 & 0 & $-1,15$ & 1,08 & 2,63 & 0,41 \\
\hline & $\mathrm{H}$ & $-149,4$ & $-0,33$ & 0,0018 & 0,0023 & 0,22 & 0 & $-0,61$ & 0,98 & 2,63 & 0,37 \\
\hline & 1 & $-136,13$ & $-0,33$ & 0,0034 & 0,0045 & 0,44 & 0 & $-1,12$ & 1,70 & 2,63 & 0,65 \\
\hline & $\mathrm{J}$ & $-148,6$ & $-0,58$ & 0,0018 & 0,0029 & 0,28 & 0 & $-0,91$ & 1,58 & 2,63 & 0,60 \\
\hline \multirow[t]{10}{*}{ 21/Jan-01/Fev } & A & $-179,51$ & 0,90 & 0,0004 & $-0,0000$ & 0,01 & 1,8 & $-0,48$ & 1,89 & 3,09 & 0,61 \\
\hline & B & $-183,34$ & 1,46 & 0,0003 & $-0,0001$ & $-0,04$ & 1,8 & $-0,18$ & 1,68 & 3,09 & 0,54 \\
\hline & C & $-183,8$ & 0,44 & 0,0003 & 0,0002 & 0,05 & 1,8 & $-1,67$ & 2,85 & 3,09 & 0,92 \\
\hline & D & $-171,09$ & 0,34 & 0,0006 & 0,0004 & 0,10 & 1,8 & $-0,90$ & 2,16 & 3,09 & 0,70 \\
\hline & $E$ & $-169,6$ & $-0,28$ & 0,0006 & 0,0008 & 0,25 & 1,8 & $-1,11$ & 2,34 & 3,09 & 0,76 \\
\hline & $\mathrm{F}$ & $-177,2$ & 0,13 & 0,0004 & 0,0004 & 0,12 & 1,8 & $-1,47$ & 2,63 & 3,09 & 0,85 \\
\hline & G & $-158,59$ & 0,80 & 0,0011 & 0,0002 & 0,06 & 1,8 & $-0,21$ & 1,63 & 3,09 & 0,53 \\
\hline & $\mathrm{H}$ & $-192,3$ & 0,39 & 0,0002 & 0,0001 & 0,04 & 1,8 & $-3,11$ & 4,06 & 3,09 & 1,31 \\
\hline & 1 & $-169,05$ & 1,66 & 0,0007 & $-0,0004$ & $-0,12$ & 1,8 & $-0,16$ & 1,73 & 3,09 & 0,56 \\
\hline & $\mathrm{J}$ & $-190,2$ & 0,18 & 0,0002 & 0,0002 & 0,06 & 1,8 & $-0,12$ & 1,56 & 3,09 & 0,50 \\
\hline \multirow[t]{10}{*}{ 07-11/Fev. } & A & $-187,68$ & 1,20 & 0,0003 & 0,0001 & 0,00 & 0,6 & $-0,69$ & 3,23 & 3,81 & 0,85 \\
\hline & B & $-161,39$ & 1,36 & 0,0010 & 0,0003 & 0,00 & 0,6 & $-1,16$ & 4,41 & 3,81 & 1,16 \\
\hline & C & $-204,8$ & 0,95 & 0,0001 & 0,0000 & 0,00 & 0,6 & $-1,29$ & 4,73 & 3,81 & 1,24 \\
\hline & D & $-177,21$ & 0,74 & 0,0004 & $-0,0001$ & $-0,01$ & 0,6 & $-1,24$ & 4,57 & 3,81 & 1,20 \\
\hline & E & $-216,5$ & 1,25 & 0,0001 & 0,0000 & 0,00 & 0,6 & $-1,38$ & 4,63 & 3,81 & 1,22 \\
\hline & $\mathrm{F}$ & $-222,6$ & 0,95 & 0,0000 & 0,0000 & 0,00 & 0,6 & $-0,46$ & 2,65 & 3,81 & 0,70 \\
\hline & G & $-139,70$ & 1,61 & 0,0029 & 0,0017 & 0,00 & 0,6 & $-0,65$ & 3,13 & 3,81 & 0,82 \\
\hline & $\mathrm{H}$ & $-222,4$ & 0,79 & 0,0000 & 0,0000 & 0,00 & 0,6 & $-1,11$ & 4,28 & 3,81 & 1,12 \\
\hline & 1 & $-193,54$ & 4,32 & 0,0002 & 0,0006 & 0,00 & 0,6 & $-1,02$ & 4,05 & 3,81 & 1,06 \\
\hline & $\mathrm{J}$ & $-223,4$ & 2,43 & 0,0000 & $-0,0001$ & $-0,01$ & 0,6 & $-1,58$ & 5,48 & 3,81 & 1,44 \\
\hline
\end{tabular}




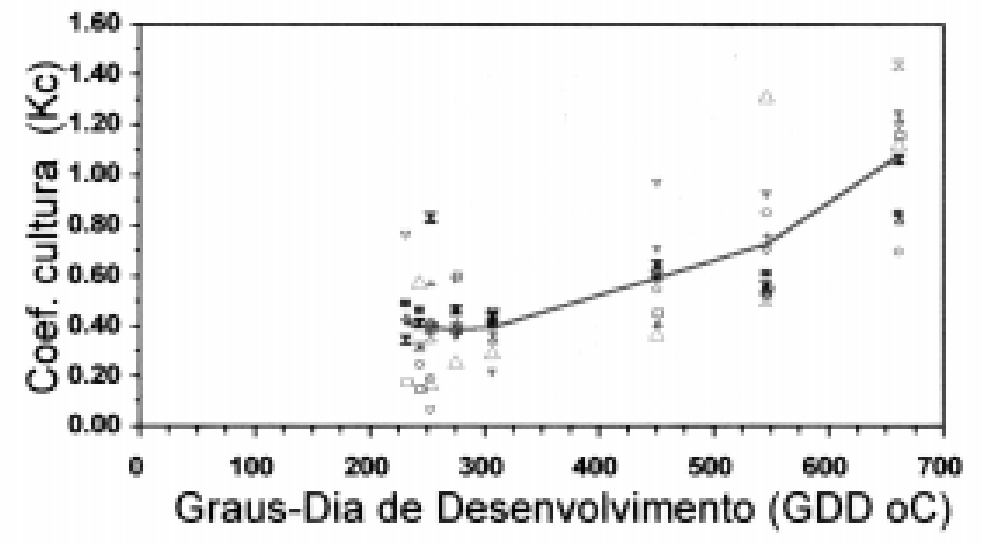

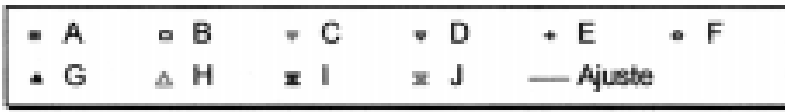

Figura 1. Coeficientes de cultura (Kc) com base em graus-dia de desenvolvimento (GDD), para as diferentes parcelas estudadas. Tucson-AZ, 1994-1995.

\section{Equação 16}

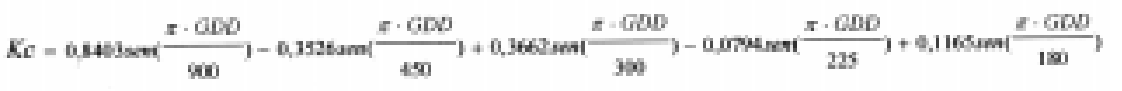

mento, sendo portanto os mesmos envolvidos na evapotranspiração máxima da cultura em estudo, a relação entre ETm e ETo não será reduzida como consequência de redução na ETo apenas.

Observou-se uma variação relativamente grande nos resultados de ETm para as diferentes parcelas dentro de um mesmo período. No primeiro período, por exemplo, os valores de ETm variaram de 0,50 a 2,20 mm/dia. Esta variação não deveria existir caso não tivessemos nenhuma diferença na capacidade de retenção de água pelo solo das diferentes parcelas, bem como diferenças na fertilidade do solo. Embora todo o cuidado tenha sido tomado para que as parcelas tivessem as mesmas características, tanto de armazenamento de água como de fertilidade, os valores de ETm não foram os mesmos. As diferenças podem ter sido devidas, também, a erros inevitáveis de determinação da tensão da água para cálculo do gradiente de potencial, causados por diferenças de leitura nos tensiômetros, ou mesmo, erros de estimativa do armazenamento de água pelo solo. Nos demais períodos de avaliação, correpondentes ao estádios de maior cobetura vegetal, as variações entre ETm foram menores com média crescentes de um período para o outro (Tabela 1).

Percebe-se uma grande variação nos resultados de Kc (Tabela 1) entre as parcelas, explicada pela variação entre os valores de ETm. Além dos inevitáveis erros na determinação de ETm pelo método do balanço hídrico do solo, temse também a variabilidade espacial das caracteristicas físicas do solo (Cassel \& Nielsen, 1986). Foi prevendo estas variações que se decidiu por trabalhar com dez parcelas ao invés de somente uma. Apesar das variações de Kc entre as parcelas, para um mesmo período, percebe-se o crescimento dos valores médios a partir do segundo período, tornando-se máximo por ocasião da colheita (Figura 1). Como Kc reflete a exigên- cia hídrica da cultura, a Figura 1 mostra também que tal exigência não é uniforme. Além dos fatores de solo que afetam a deteminação de ETm pelo método usado neste estudo, tem-se também o fator fisiologia da planta. A natural desuniformidade entre área foliar das plantas nas diferentes parcelas acarreta também variações de ETm e consequentemente de $\mathrm{Kc}$.

Os valores de graus-dia, GD, foram calculados usando se as equações 10, 11, 12 e 13. Vale lembrar que GDD corresponde ao acumulo de GD a partir da data da germinação que ocorreu, de maneira geral, em 09 de novembro de 1994 (Tabela 2). Os dados usados para obtenção de GD, foram: temperatura máxima e temperatura mínima do dia, registradas no local do experimento, e temperaturas cardeais dadas por Madariaga \& Knott (1951) e Slack et al. (1994).

A partir das Tabelas 1 e 2, obteve-se os valores de Kc como função de GDD, representados na Figura 1, considerandose a data média de cada período estudado.

A Figura 1 mostra o ajuste obtido, pelo método de regressão linear múltipla, segundo metodologia proposta por Fox Jr. et al.(1992) usando séries de Fourier para descrever Kc com base em GDD. A equação obtida, com $r^{2}=0.9951$, no ajuste foi:

(Equação 16)

Existe uma boa correlação entre grausdia de desenvolvimento (GDD) e consumo relativo $(\mathrm{Kc})$ da cultura da alface, representada pela série seno de Fourier, podendo a equação de Kc como função de GDD ser usada em projetos ou manejo de irrigação de alface do tipo folhosa.

O maior consumo relativo de água na cultura da alface ocorreu na ocasião da colheita. Portanto, o valor de Kc a ser usado na elaboração de um projeto de irrigação desta cultura deverá ser o máximo obervado, porém, este deverá ser usado somente durante o período de colheita.

Os coeficientes $\left(c_{i}\right)$ da equação obtida podem ser inseridos no programa de manejo de irrigação AZSCHED de Fox Jr. et al. (1992), para um GDD final de $900^{\circ} \mathrm{C}$, de maneira a se usar os coeficientes de cultura específicos para a alface do tipo folhosa. Para os não usuários do programa, sugere-se os valo- 
Coeficientes de cultura e graus-dia em alface.

Tabela 2. Valores de GD e GDD a partir da data de germinação da alface. Tucson-AZ, 1994-1995.

\begin{tabular}{|c|c|c|c|c|c|c|c|c|}
\hline Data & GD & GDD & Data & GD & GDD & Data & GD & GDD \\
\hline & ${ }^{\circ} \mathrm{C}$ & ${ }^{\circ} \mathrm{C}$ & & ${ }^{\circ} \mathrm{C}$ & ${ }^{\circ} \mathrm{C}$ & & ${ }^{\circ} \mathrm{C}$ & ${ }^{\circ} \mathrm{C}$ \\
\hline 09/Nov/94 & 9,23 & 9,23 & 13/Dez & 6,28 & 244,82 & 16/Jan & 3,91 & 486,69 \\
\hline $10 /$ Nov & 11,39 & 20,62 & 14/Dez & 4,90 & 249,72 & 17/Jan & 1,02 & 487,71 \\
\hline 11/Nov & 10,71 & 31,33 & 15/Dez & 4,41 & 254,13 & 18/Jan & 3,42 & 491,13 \\
\hline $12 / \mathrm{Nov}$ & 9,76 & 41,09 & 16/Dez & 5,85 & 259,98 & 19/Jan & 5,61 & 496,74 \\
\hline $13 /$ Nov & 7,01 & 48,10 & 17/Dez & 7,99 & 267,97 & 20/Jan & 7,53 & 504,27 \\
\hline $14 /$ Nov & 6,00 & 54,10 & 18/Dez & 8,79 & 276,76 & 21/Jan & 4,82 & 509,09 \\
\hline $15 /$ Nov & 8,03 & 62,13 & 19/Dez & 7,72 & 284,48 & 22/Jan & 5,32 & 514,41 \\
\hline $16 /$ Nov & 11,96 & 74,09 & 20/Dez & 7,42 & 291,90 & 23/Jan & 6,95 & 521,36 \\
\hline $17 /$ Nov & 6,40 & 80,49 & 21/Dez & 8,37 & 300,27 & 24/Jan & 9,30 & 530,66 \\
\hline 18/Nov & 7,18 & 87,67 & 22/Dez & 8,04 & 308,31 & 25/Jan & 10,71 & 541,37 \\
\hline 19/Nov & 2,28 & 89,95 & 23/Dez & 11,51 & 319,82 & 26/Jan & 5,96 & 547,33 \\
\hline $20 /$ Nov & 3,07 & 93,02 & 24/Dez & 9,66 & 329,48 & 27/Jan & 4,19 & 551,52 \\
\hline $21 /$ Nov & 6,11 & 99,13 & 25/Dez & 9,91 & 339,39 & 28/Jan & 4,92 & 556,44 \\
\hline $22 /$ Nov & 6,31 & 105,44 & 26/Dez & 4,31 & 343,70 & 29/Jan & 5,41 & 561,85 \\
\hline 23/Nov & 12,00 & 117,44 & 27/Dez & 6,76 & 350,46 & 30/Jan & 6,30 & 568,15 \\
\hline $24 /$ Nov & 10,34 & 127,78 & 28/Dez & 6,16 & 356,62 & 31/Jan & 7,22 & 575,37 \\
\hline $25 /$ Nov & 9,06 & 136,84 & 29/Dez & 6,27 & 362,89 & 01/Fev/95 & 8,43 & 583,80 \\
\hline $26 /$ Nov & 0,00 & 136,84 & 30/Dez & 3,27 & 366,16 & $02 / F e v$ & 9,70 & 593,50 \\
\hline $27 /$ Nov & 2,39 & 139,23 & $31 / \mathrm{Dez}$ & 4,31 & 370,47 & 03/Fev & 10,27 & 603,77 \\
\hline 28/Nov & 3,14 & 142,37 & 01/Jan/95 & 5,39 & 375,86 & 04/Fev & 10,45 & 614,22 \\
\hline $29 /$ Nov & 4,94 & 147,31 & 02/Jan & 7,66 & 383,52 & 05/Fev & 8,86 & 623,08 \\
\hline $30 /$ Nov & 7,06 & 154,37 & 03/Jan & 6,36 & 389,88 & 06/Fev & 8,95 & 632,03 \\
\hline 01/Dez/94 & 8,34 & 162,71 & 04/Jan & 6,11 & 395,99 & 07/Fev & 9,47 & 641,50 \\
\hline 02/Dez & 8,23 & 170,94 & 05/Jan & 8,01 & 404,00 & 08/Fev & 10,37 & 651,87 \\
\hline 03/Dez & 8,08 & 179,02 & 06/Jan & 5,57 & 409,57 & 09/Fev & 10,20 & 662,07 \\
\hline 04/Dez & 8,43 & 187,45 & 07/Jan & 7,01 & 416,58 & 10/Fev & 6,01 & 668,08 \\
\hline 05/Dez & 8,56 & 196,01 & 08/Jan & 8,94 & 425,52 & $11 /$ Fev & 7,06 & 675,14 \\
\hline 06/Dez & 9,11 & 205,12 & 09/Jan & 9,05 & 434,57 & $12 / \mathrm{Fev}$ & 8,31 & 683,45 \\
\hline 07/Dez & 8,86 & 213,98 & 10/Jan & 9,40 & 443,97 & $13 / \mathrm{Fev}$ & 11,06 & 694,51 \\
\hline 08/Dez & 5,81 & 219,79 & 11/Jan & 7,86 & 451,83 & 14/Fev & 10,66 & 705,17 \\
\hline 09/Dez & 3,60 & 223,39 & 12/Jan & 7,76 & 459,59 & 15/Fev & 8,51 & 713,68 \\
\hline 10/Dez & 4,80 & 228,19 & 13/Jan & 7,11 & 466,70 & $16 / F e v$ & 9,71 & 723,39 \\
\hline 11/Dez & 5,11 & 233,30 & 14/Jan & 8,12 & 474,82 & 17/Fev & 9,24 & 732,63 \\
\hline 12/Dez & 5,24 & 238,54 & 16/Jan & 7,96 & 482,78 & 18/Fev & 9,78 & 742,41 \\
\hline
\end{tabular}

res de Kc, para todo o ciclo, como função do acumulo de unidades térmicas, apresentados na Tabela 3.

A Tabela 3 permite definir a quantidade de água a ser reposta em cada irrigação, calculando a ETm a partir da ETo do local, usando os valores de Kc de acordo com os valores de GDD calculados desde a emergência.

Sugere-se que os dados obtidos de Kc como funcão de GDD sejam testados em diferentes tipos de clima e épocas de plantio, bem como em diferentes solos, de modo a confirmar ou rejeitar a idéia de extrapolação de informações necessária no manejo de irrigação da cultura de alface. De qualquer modo, Kc como função de GDD com certeza aproxima-se mais da realidade quando comparado com os valores existentes em tabelas, de Kc como função de dias após germinação, que não informam as condições em que tais dados foram obtidos. 
Tabela 3. Sugestão de coeficientes de cultura para os diferentes estádios vegetativos da alface do tipo folhosa. Tucson-AZ, 1995-1996.

\begin{tabular}{cc}
\hline GDD (oC) & Kc \\
\hline até 300 & 0,40 \\
300 a 350 & 0,45 \\
350 a 400 & 0,50 \\
400 a 450 & 0,60 \\
450 a 500 & 0,65 \\
500 a 550 & 0,75 \\
550 a 600 & 0,85 \\
600 a 650 & 1,00 \\
650 a 750 & 1,20 \\
\hline
\end{tabular}

\section{LITERATURA CITADA}

CASSEL, D.K.; NIELSEN, D.R. Field Capacity and Available Water Capacity. In: A. Klute (ed.). Methods of Soil Analysis, Part 1. $2^{\text {nd }}$ ed Agron. Monogr. 9. ASA and SSSA, Madison, WI. p. 901-926, 1986

CUENCA, R.H. Irrigation System Design - An Engineering Approach. Prentice-Hall Inc., Englewood Cliffs, New Jersey, 1989. 552 p.

DOORENBOS, J.; PRUITT, W.O. Guidelines for predicting crop water requirements. Rome, FAO, 1977. 179 p. (FAO - Irrigation and Drainage Paper, 24)

ERIE, L.J.; FRENCH,O.F.; BUCKS,D.A.; HARRIS,K. Consumptive Use of Water by Major Crops in the Southwestern United States. USDA Agricultural Research Service, Conservation Research Report 29, 1982. 42 p.

FERVERT, D.K.; HILL, R.W.; BRAATEN, B.C. Estimation of FAO Evapotranspiration Coefficients. Journal of the Irrigation and Drainage Division, Am. Society of Civil Engineers, v. 109, n. 2, p. 265-269, 1983.

FOX Jr., F.A.; SHERER, T.; SLACK, D.C.; CLARK, L.J. Arizona Irrigation Scheduling- AZSCHED ver. 1.01-Users Manual. The University of Arizona/Cooperative Extension/Agricultural and Biosystems Engineering, 1992. 36 p.
GILMORE, E; ROGERS, J.S. Heat Units as a Method of Measuring Maturity in Corn. Agronomy Journal, Madison, v. 50,. p. 611$615,1958$.

INFELD, J.A.; SILVA, J.B. da. Somas térmicas da duração da fase vegetativa do arroz irrigado. (Oryza sativa. L.) In: CONGRESSO BRASILEIRO DE AGROMETEOROLOGIA, 5․ BelémPA, Anais... Belém, SBA, 1987. p. 160-161.

JENSEN, M.E.; BURMAN, R.D.; ALLEN, R.G. (ed.) Evapotranspiration and Irrigation Water Requirements. American Society of Civil Engineers - ASCE, New York, NY, 1990. 332 p.

JURY, W.A.; GARDNER, W.R.; GARDNER, W.H. Soil Physics. John Wiley \& Sons, Inc., New York, NY, 1991. 328 p.

KHAN, A.A. Field evaluation of water and solute movement from a point source. Tucson. The University of Arizona, 1994. 224 p. (Ph.D. dissertation)

MADARIAGA, F.J.; KNOTT, J.E. Temperature summations in relation to lettuce growth. Proceedings of the American Society of Horticulture Science., v. 58, p. 147-152, 1951.

MOTA, FS. Meteorologia Agrícola. 7a ed. São Paulo, Nobel, 1986. 376 p.

PLANT, RE.; HORROCKS, RD.; GRIMES, DW.; ZELINSKI, LJ. CALEX/cotton: an integrated expert system application for irrigation scheduling. Transaction of the ASAE, v. 35, p. 1833-1838, 1992.
REICHARDT, K. Processos de Transferência no Sistema Solo-Planta-Atmosfera. 4a ed., Campinas-SP, Fundação Cargill, 1985. 448 $\mathrm{p}$.

SAMMIS, T.W. Comparison of sprinkler, trickle, subsurface, and furrow irrigation methods for row crops. Agronomy Journal, v. 72, n. 5, p. 701-704, 1980.

SAMMIS, T.W.; MAPEL, C.L.; LUGG, D.G.; LANSFORD, R.R.; McGUCKIN, J.T. Evapotranspiration crop coefficients predicted using growing-degree-days. Transactions of the ASAE, v. 28, n. 3, p. 773-780, 1985.

SAMMIS, T.W.; KRATKY, B.A.; WU, I.P. Effects of limited irrigation on lettuce and chinese cabbage yields. Irrigation Science, v. 9, p.187198, 1988.

SHAYYA, E.H.; BRALTZ, V.F.; LOUDON, T.L. Irrigation scheduling based on growing degree days. ASAE Paper 91-2643. Winter Meeting, 1991. 23 p.

SILVA, E.L. da. Irrigation methods and mangement effects on leaf lettuce (L. sativa, L.) water use and nitrogen leaching. Tucson: The University of Arizona, 1995. 143 p. (Ph.D. dissertation)

SLACK, D.C.; FOX Jr., F.A.; MARTIN, E.C.; CLARK, L.J. Growing-degree-day based crop coefficients for irrigation management. In: CONGRESSO NATIONAL ASOCIACION MEXICANA DE INGENIERIA AGRICOLA, 4, Cuautitlán Izcalli, Estado de México, México, 1994. Anais... p. 7-13.

SLATYER, R.O. Plant-Water Relationships. Academic Press, New York, NY, 1970. 366 p.

SNYDER, R.L. Hand calculting degree days. Journal of Agriculture and Forest Meteorology, v. 35, p. 353-358, 1985.

TAYLOR, S.A. Managing Irrigation Water on the Farm. Transactions of the ASAE, v. 8, n. 3, p. 433-437, 1965.

TOSSO, J.T.; TORRES, J.J. Relaciones hidricas de la vid, bajo diferentes niveles de riego usando goteo, aspersion y surcos. I. Evapotranspiracion y eficiencia en el uso del agua. Agricultura Tecnica, v. 46, p. 193-198, 1986. 